\title{
Correction to: Graphical translators for mean curvature flow
}

\author{
D. Hoffman ${ }^{1} \cdot$ T. Ilmanen ${ }^{2} \cdot$ F. Martín ${ }^{3} \cdot$ B. White ${ }^{1}$
}

Published online: 7 August 2019

(c) Springer-Verlag GmbH Germany, part of Springer Nature 2019

\section{Correction to: Calc. Var. (2019) 58:117 https://doi.org/10.1007/s00526-019-1560-x}

In the published article, the reference cited at the end of the proof of Theorem 5.1 was published incorrectly. The relevant line should read as: "It follows by Morse Theory (see Theorem 7.1(17) in [8]) that $F$ has at most one interior critical point, and it is a simple critical point."

Publisher's Note Springer Nature remains neutral with regard to jurisdictional claims in published maps and institutional affiliations.

The original article can be found online at https://doi.org/10.1007/s00526-019-1560-x.

F. Martín

fmartin@ugr.es

D. Hoffman

dhoffman@stanford.edu

T. Ilmanen

tom.ilmanen@math.ethz.ch

B. White

bcwhite@stanford.edu

1 Department of Mathematics, Stanford University, Stanford, CA 94305, USA

2 Department of Mathematics, E. T. H. Zürich, Rämistrasse 101, 8092 Zürich, Switzerland

3 Departmento de Geometría y Topología, Instituto de Matemáticas IE-Math Granada, Universidad de Granada, 18071 Granada, Spain 